\section{THE ROLE OF VARIOUS PREDICTORS OF SEIZURE RECURRENCE IN ASSIGNMENT OF YOUNG MEN TO PROFESSIONS WITH ASSOCIATED EXPOSURE TO (SEIZURE) RISK FACTORS}

1,2 Michal Tavor, ${ }^{3}$ Miri Y Neufeld, ${ }^{2,4}$ Gabriel Chodick, ${ }^{2,5}$ Oren Zack, ${ }^{1,2}$ Shlomo Moshe. ${ }^{1}$ Maccabi Healthcare Services, The Occupational Clinic, Holon, Israel; ${ }^{2}$ Sackler Faculty of Medicine, School of Public Health, Department of Environmental and Occupational Medicine, Tel Aviv, Israel; ${ }^{3}$ EEG and Epilepsy Unit, Tel Aviv, Israel; ${ }^{4}$ Maccabi Healthcare Services, Central Headquarter, Tel Aviv, Israel, Tel Aviv, Israel; ${ }^{5}$ Israel Defence Force, Medical Corps, Israel, Ramat Gan, Israel

\subsection{6/oemed-2014-102362.309}

Objectives To study the risk of epileptic seizures as a function of disease severity and occupational stress (physical and mental) in new military recruits in the Israel Defense Forces (IDF).

Method The medical records of over 145000 18-year old men, recruited to the IDF between the late-nineties and early twothousands, were used to assemble a cohort, which was followed for a period of 36 months. The severity of the disease was determined according to 5 categories. Recruits were subdivided according to the following occupational categories: Combat Units (CU), Maintenance Units (MU) and Administrative Units (AU).

Results The annual incidence rate for a first seizure was 26/100 000. The rates in $\mathrm{CU}$ and $\mathrm{MU}$ were lower than $\mathrm{AU}$ (0.41 and 0.81 vs. 1 respectively, $\mathrm{p}<0.01)$. Similar findings were found in other disease categories.

Conclusions The low rate for a first seizure and the lower overall seizure rate in $\mathrm{CU}$ compared to $\mathrm{MU}$ and $\mathrm{AU}$ may be explained by the recruiting of a healthy population, higher motivation than before, and meticulous adherence to diagnostic criteria. The higher recurrence rate in our research as compared to the previous follow up, may be attributable to the modification of disease categories. Our findings suggest moderating occupational restrictions for epilepsy patients and using EEG and relapse-free periods of 2-6 years as fitness for work criteria. We propose the reassessment of severity criteria currently used by the IDF.

\section{ENVIRONMENTAL EXPOSURE TO NANOPARTICLES IN SARDINIA, ITALY: A PILOT STUDY OF RESIDENTIAL EXPOSURE NEARBY AN INDUSTRIAL AREA AND A MILITARY SHOOTING RANGE}

Marcello Campagna, Gabriele Marcias, Natalia Angius, Daniele Fabbri, Marcello Noli, Sergio Pili, Ilaria Pilia, Giuseppe Avataneo, Pierluigi Cocco. University of Cagliari, Monserrato, Cagliari, Italy

\subsection{6/oemed-2014-102362.310}

Objectives Objective of our pilot study was to explore the airborne ultrafine particle count in residential areas nearby industrial and military settings with reference to urban and rural areas.

Method We monitored airborne ultrafine (ranging 7nm - 10 microm) particles in residential areas nearby a large oil refinery, a military shooting range, in the largest urban area in the region and in a rural area. We conducted eight samplings $(6 \mathrm{~h}$ each) using a Electrical low pressure impactor (ELPI plus Dekati, Tampere, FInland). Wind speed and direction, temperature and humidity during each sampling were registered. Data on other potential sources of ultrafine particles, from both anthropic and natural origin, were also resigeterd. The airborne nanoparticle concentration was expressed as particle count $/ \mathrm{cm}^{3}$.

Results The median ultrafine particle count was 7408 (max $179605) / \mathrm{cm}^{3}$ in the residential area nearby the oil refinery, 9079 $(\max 114281) / \mathrm{cm}^{3}$ nearby the military shooting range, 19040 $(\max 142324) / \mathrm{cm}^{3}$ in the urban area and $25419(\max 373434)$ in the rural area.

Conclusions Our results show that ultrafine particles were ubiquitous in the sampling sites. Median counts were higher in the rural area than nearby industrial and military settings. We speculate that anthropic activities, including widespread use of wood burning fireplaces in rural areas, as well as technical measures to control industrial particulate emissions implemented in the past years, might have contributed. Further studies and additional sampling will allow a more detailed picture of exposure levels to better characterise risk of possible adverse health outcomes associated with environmental exposure to nanoparticles.

\section{HEAD AND NECK CANCER AND OCCUPATIONAL EXPOSURE TO CHLORINATED SOLVENTS: RESULTS FROM THE ICARE STUDY}

${ }^{1}$ Aurore Fayossé, ${ }^{2}$ Gwenn Menvielle, ${ }^{2}$ Diane Cyr, ${ }^{2}$ Marie Sanchez, ${ }^{2}$ Isabelle Stucker, ${ }^{1}$ Danièle Luce. 'INSERM U1085, Pointe À Pitre, Guadeloupe, France; '2INSERM U1018, Villejuif, France

10.1136/oemed-2014-102362.311

Objectives To investigate the associations between head and neck cancer risk and occupational exposure to chlorinated solvents.

Method ICARE is a population based case-control study conducted in France. Analyses were restricted to men and included 1833 cases of head and neck squamous cell carcinomas (HNSCC) and 2747 controls. Complete occupational history was collected. Job-exposure matrices allowed to assess exposure to five chlorinated solvents (trichloroethylene, perchloroethylene, methylene chloride, chloroform and carbon tetrachloride). Odds ratios (ORs) adjusted for smoking, alcohol drinking and other potential confounders and 95\% confidence intervals (CI) were estimated with logistic models.

Results No association was found for occupational exposure to trichloroethylene, methylene chloride, chloroform and carbon tetrachloride, and no dose-response relationships were observed. A non-significantly increased risk of HNSCC was observed for perchloroethylene $(\mathrm{OR}=2.1$, CI 0.7-6.3), when comparing the highest tertile of cumulative exposure with no exposure. Analysis by cancer site showed that this increased risk was limited to laryngeal cancer. The risk of laryngeal cancer increased with cumulative exposure to perchloroethylene ( $\mathrm{p}$ for trend $=0,03$ ), with a significantly elevated OR $(\mathrm{OR}=5.0, \mathrm{CI} 1.6-15.6)$ for the highest tertile of cumulative exposure. Exposure to perchloroethylene was not associated with the risk of oral or pharyngeal cancer. No associations were found between other chlorinated solvents and any of the cancer sites.

Conclusions These findings suggest that high levels of exposure to perchloroethylene may increase the risk of laryngeal cancer. Our study does not provide evidence that other chlorinated solvents are risk factors for HNSCC. 